\title{
Combined Effect of Bilateral Ovariectomy and Anterior Cruciate Ligament Transection With Medial Meniscectomy on the Development of Osteoarthritis Model
}

\author{
Jae Lim Kim, MD, Chang Won Moon, MD, Young Suk Son, MD, Sang Jun Kim, MD, PhD
}

Department of Physical and Rehabilitation Medicine, Samsung Medical Center, Sungkyunkwan University School of Medicine, Seoul, Korea

\begin{abstract}
Objective To investigate the combined effect of bilateral ovariectomy (OVX) and anterior cruciate ligament transection (ACLT) with medial meniscectomy (MM) on the development of osteoarthritis (OA).

Methods Twenty female 15-week-old Sprague-Dawley rats were used. Five rats in each group underwent bilateral OVX (OVX group), bilateral ACLT with MM (ACLT with MM group), bilateral OVX plus ACLT with MM (OVX plus ACLT with MM group), and sham surgery (SHAM group). All the rats were subjected to treadmill running for 4 weeks. The behavioral evaluation for induction of OA used the number of rears method, and this was conducted at 1,2 , and 4 weeks post-surgery. Bone mineral density (BMD) was calculated with micro-computerized tomography images and the modified Mankin's scoring was used for the histological changes.

Results The number of rears in the OVX plus ACLT with MM group decreased gradually and more rapidly in the ACLT with MM group. Histologically, the OVX plus ACLT with MM group had a significantly higher modified Mankin's score than the OVX group ( $\mathrm{p}=0.008)$ and the SHAM group $(\mathrm{p}=0.008)$. BMDs of the OVX plus ACLT with MM group were significantly lower than the SHAM group ( $\mathrm{p}=0.002)$, and the ACLT with MM group ( $\mathrm{p}=0.003)$.

Conclusion We found that bilateral OVX plus ACLT with MM induced definite OA change in terms of histology and BMD compared to bilateral OVX and ACLT with MM alone. Therefore, OVX and ACLT with MM was an appropriate degenerative $\mathrm{OA}$ rat model.
\end{abstract}

Keywords Osteoarthritis, Ovariectomy, Anterior cruciate ligament, Meniscus, Animal model

Received September 3, 2015; Accepted October 28, 2015

Corresponding author: Sang Jun Kim

Department of Physical and Rehabilitation Medicine, Samsung Medical Center, Sungkyunkwan University School of Medicine, 81 Irwon-ro, Gangnamgu, Seoul 06351, Korea. Tel: +82-2-3410-6069, Fax: +82-2-3410-0052, E-mail: guitarren.kim@samsung.com

ORCID: Jaelim Kim (http://orcid.org/0000-0001-8267-2748); Chang Won Moon (http://orcid.org/0000-0003-4096-7470); Young Suk Son (http://orcid. org/0000-0003-1328-4631); Sang Jun Kim (http://orcid.org/0000-0002-0479-7526).

(c) This is an open-access article distributed under the terms of the Creative Commons Attribution Non-Commercial License (http://creativecommons.org/ licenses/by-nc/4.0) which permits unrestricted noncommercial use, distribution, and reproduction in any medium, provided the original work is properly cited. Copyright (c) 2016 by Korean Academy of Rehabilitation Medicine 


\section{INTRODUCTION}

Osteoarthritis (OA) is characterized by a progressive loss of articular cartilage and defined as the presence of osteophytes in radiographic findings [1]. There have been many preclinical and clinical trials in order to study and manage the symptoms of $\mathrm{OA}$ and to delay its progression. However, there have been mismatches between preclinical and clinical results, which might be caused by the differences between the surgically or chemically induced $\mathrm{OA}$ animal models and the degenerative $\mathrm{OA}$ in patients [2]. To overcome this problem, development of the best suited animal OA models is mandatory. Several methods including genetic modification (STR/ort mice), anterior cruciate ligament transection (ACLT), medial meniscectomy (MM), intra-articular monosodium iodoacetate injection, and bilateral ovariectomy (OVX) have been developed as OA animal models. Recommendations for the use of preclinical models in various situations were done in a previous study [3]. This was part of an effort to develop more effective drugs from preclinical work by reviewing the pathogenesis of various $\mathrm{OA}$ animal models.

There is, however, no preclinical model that represents the clinical aspects of the degenerative OA in humans. This may be because the pathophysiology of the OA in humans has not yet been fully elucidated, and most animals usually used in laboratory settings crawl on four limbs rather than walk bipedally. In addition, degenerative OA in humans is usually brought about by a combination of many factors, such as genetics, biomechanics, hormonal changes, and traumatic history. As such, preclinical models made by one intervention are insufficient to simulate the human OA contributed by multiple factors.

Anterior cruciate ligament transection and MM are generally used for the OA animal model because these procedures evoke joint instability associated with mechanical stress. This surgical model causes not only molecular changes in the cartilage but also results in synovial inflammation and subchondral bone sclerosis like human OA [4]. However, these changes are not continuously aggravated as the time is elapsed, which is different from human OA [4]. A systematic review of OA animal models elucidated the detrimental effect of ovariectomy on the cartilage, which was mediated by matrix metalloproteases, nitric oxide, and interleukins [5]. Consider- ing that $\mathrm{OA}$ incidence in women increases abruptly after menopause, bilateral OVX will play an important role in mimicking the development of the OA changes in postmenopausal women [6].

Although ACLT with MM or OVX are generally used for production of OA animal model, the combined effect of these two techniques on the development of OA has not yet been elucidated. We thought that the combination of these two techniques may mimic a better model of human OA than one technique alone. Mechanical stress with joint instability caused by ACLT with MM will affect the destruction of cartilage as ACLT with MM causes joint instability. Also, bilateral OVX will affect the composition of cartilage because animals with bilateral OVX have been shown to have a low aggregate and shear modulus in their cartilage [7]. Therefore, we sought to investigate the combined effect of ACLT with MM and bilateral OVX on development of $\mathrm{OA}$ in rats.

\section{MATERIALS AND METHODS}

A total of 20 female 15-week-old Sprague-Dawley rats were used for this study. All rats were kept in cages located in an air-conditioned room, while commercial pellet and tap water were provided ad libitum. After one week of acclimation, the following experimental groups were developed: (1) 5 rats with bilateral OVX (OVX group), (2) 5 rats with bilateral ACLT with MM (ACLT with MM group), (3) 5 rats with bilateral OVX plus ACLT with MM (OVX plus ACLT with MM group), and (4) 5 rats with sham surgery (SHAM group). All the rats were subjected to treadmill running with a speed of $17 \mathrm{~m} / \mathrm{min}$ for 20 $\mathrm{min} /$ day, 5 days/week for 4 weeks. Behavioral tests were conducted 1,2, and 4 weeks after the induction of OA. After the end of the behavioral tests, all the rats were euthanized and their knee joints were extracted. Microcomputerized tomography (CT) and histologic studies were then performed.

\section{Preparation of OVX}

For the OVX group, the rats were anesthetized with an intra-muscular injection of a 1:1 mixture of tiletamine and zolazepam (Virbac, Carros, France) with xylazine (Bayer Vital GmbH, Leverkusen, Germany) at a dose of 30 mg zolazepam and $10 \mathrm{mg}$ xylazine per $\mathrm{kg}$ of body weight. Placed in dorsal recumbency, the ventral mid-lumbar 
area was shaved and swabbed with $70 \%$ ethyl alcohol. A $2-3 \mathrm{~cm}$ ventral midline skin incision was made at the level of the uterus, followed by a $2-\mathrm{cm}$ midline muscle incision. After pulling aside the muscle and fascia, left side ovarian duct was found and traced to the ovary. After the ligation of the ovarian duct at $1 \mathrm{~cm}$ distal to the ovary, the ovarian duct and peritoneum attached to the ovary were cut by a scissor and the ovary was extracted (Fig. 1). The other side with the right-side ovary underwent the same procedure and the muscle and skin from the incision were then sutured back together layer by layer.

\section{Preparation of ACLT with MM}

After the same anesthetic procedure as for previous group, the anterior surface of the left hind limb was shaved and the skin around the incision area was cleansed with $70 \%$ ethyl alcohol. The skin and fascia on the knee cap region of the left hind limb was vertically incised in the midline for a distance of approximately $4 \mathrm{~cm}$. The patella was pushed laterally to expose the articular cavity. The synovial membrane was excised and the knee joint was bent to expose the anterior cruciate ligament. Then, the anterior cruciate ligament was transected and the medial meniscus was completely removed with a surgical scissor (Fig. 2). The patella was then relocated
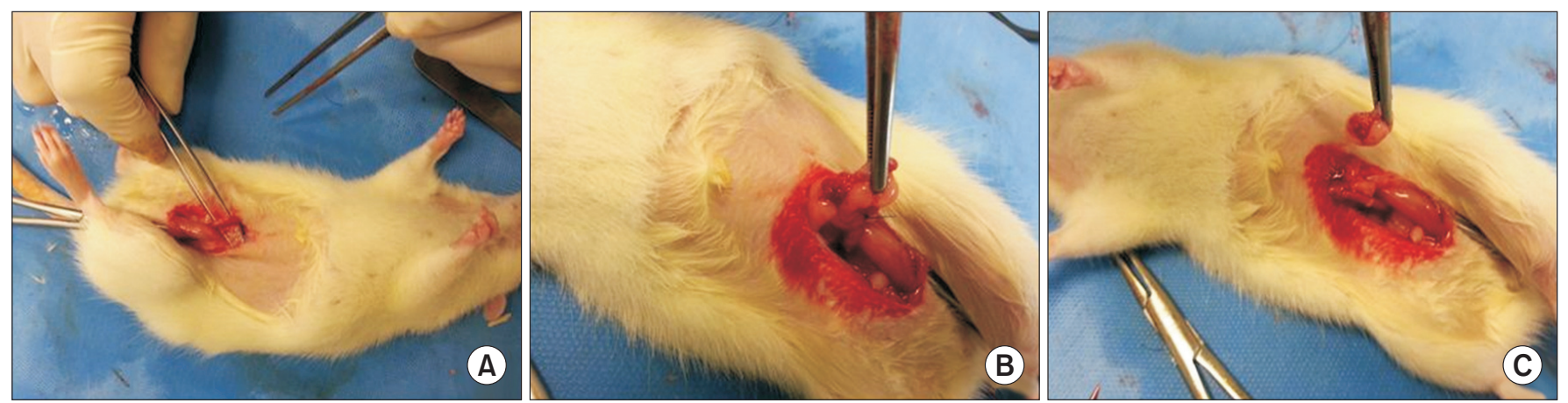

Fig. 1. Details shown for the ovariectomy procedure. (A) A 2- to 3-cm ventral midline skin incision and a 2-cm midline muscle incision were made at the level of uterus. (B) After the ligation of the ovarian duct, (C) the ovary was extracted.
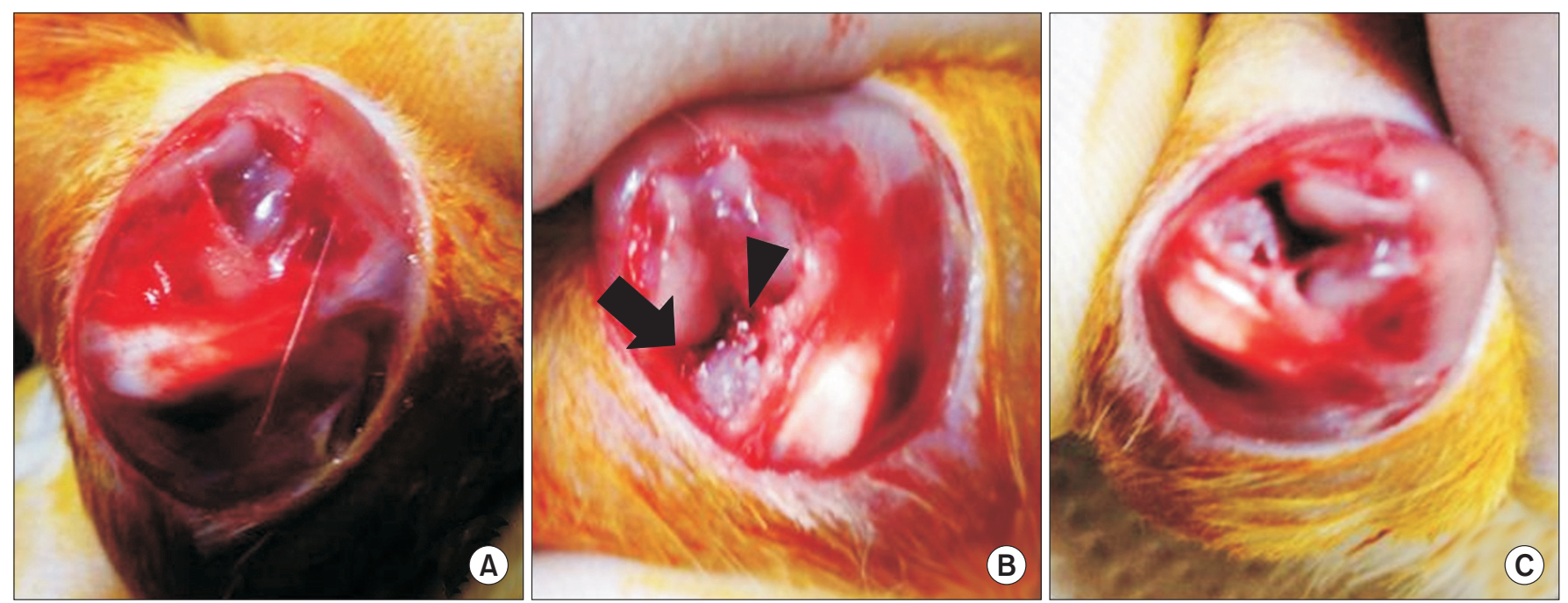

Fig. 2. (A) A 4-cm vertical midline incision was made in the skin and fascia on the knee cap region of the left hind limb.

(B) The patella was pushed laterally and the synovial membrane was excised and the knee joint was bent to expose the anterior cruciate ligament (solid triangle) and the medial meniscus (solid arrow). (C) The anterior cruciate ligament was transected and the medial meniscus was completely removed. 
back to its original position, and the fascia and skin were closed with 3-0 polydioxanone suture. The other limb underwent the same procedure.

\section{Preparation of SHAM surgery}

After the same anesthetic procedure, the skin and fascia on the knee cap region of the bilateral hind limb were vertically incised in the midline for a distance of approximately $4 \mathrm{~cm}$. Without additional intervention, the fascia and skin were closed with 3-0 polydioxanone suture.

\section{Evaluation of behavioral tests (number of rears)}

The number of rears is a common measure of activity and exploratory behavior and has been widely applied in the evaluation of movement in various rat model studies $[8,9]$. Also, this method was modified from the method used in a previous study [10]. An open field acrylic box with a dimension of $30 \mathrm{~cm} \times 30 \mathrm{~cm} \times 30 \mathrm{~cm}$ was placed in a room with minimum noise. Foam stamp pads were placed on the floor of the box and 4 pieces of white papers were attached to 4 sides of the box. Individual rats were placed in the box and left there for 30 minutes. The rats got their paws covered in foam stamp pad and left footprints on the paper when they stood on their hind limbs and touched the walls of the box with their fore limbs. The papers with footprints were collected after the test. The scanned images of the papers were analyzed with a medical imaging software program, ImageJ ver. 1.45 (National Institutes of Health, Bethesda, MD, USA). The number of distinct particles above $5 \mathrm{~cm}$ from the bottom of the paper was counted by the software. The number of rears was estimated by dividing the number of particles by 14, which was derived from the fact that there are 7 distinguishable particles found per footprint and 2 footprints per rear (Fig. 3). The evaluation was done at 1 , 2 , and 4 weeks after the surgical operations.

\section{Histologic study}

For the histologic study, knee joint tissues with synovial membrane were extracted by trimming the muscles. They were fixed in $10 \%(\mathrm{w} / \mathrm{v})$ buffered formalin, decalcified by hydrogen chloride/EDTA solution, and embedded in paraffin and then sectioned in the sagittal plane under the midline at $6 \mu \mathrm{m}$ thickness. Collagen was stained using Masson's trichrome and sulfated glycosaminoglycan was stained with Safranin O. To observe the nucleus and cytoplasm, hematoxylin and eosin (H\&E) staining was also performed. The light microscopic photographs of stained slides (at $\times 40$ and $\times 200$ ) were taken.

The modified Mankin's scoring system is one of the histologic and histochemical grading systems used to quantify the degree of $\mathrm{OA}[11]$. Items for scoring were (i) cartilage structure (0-6), (ii) cartilage cells (0-3), (iii) Safranin $O$ staining (0-4), and (iv) tidemark integrity (0-1). The modified Mankin's score range is from 0 to 14 and the higher the score, the more severe is the extent of osteoarthritis $[12,13]$.

\section{Micro-CT imaging study}

Knee joint tissues of rats after removing synovial membrane and the muscles were imaged using a micro-CT scanner after a month following the OA surgical induc-

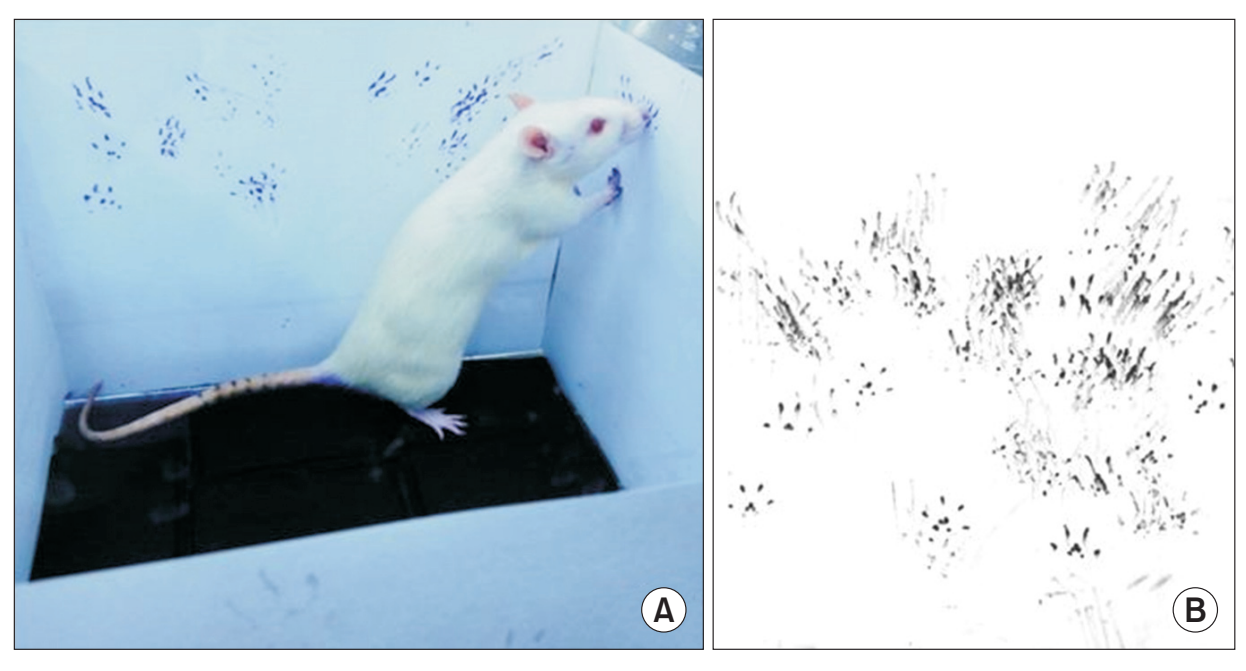

Fig. 3. Measurement of the number of rears. (A) With paws covered in foam stamp pad, the rats stood on their hind limbs and touched the walls of the box with their forelimbs. (B) The footprints left on the paper. 

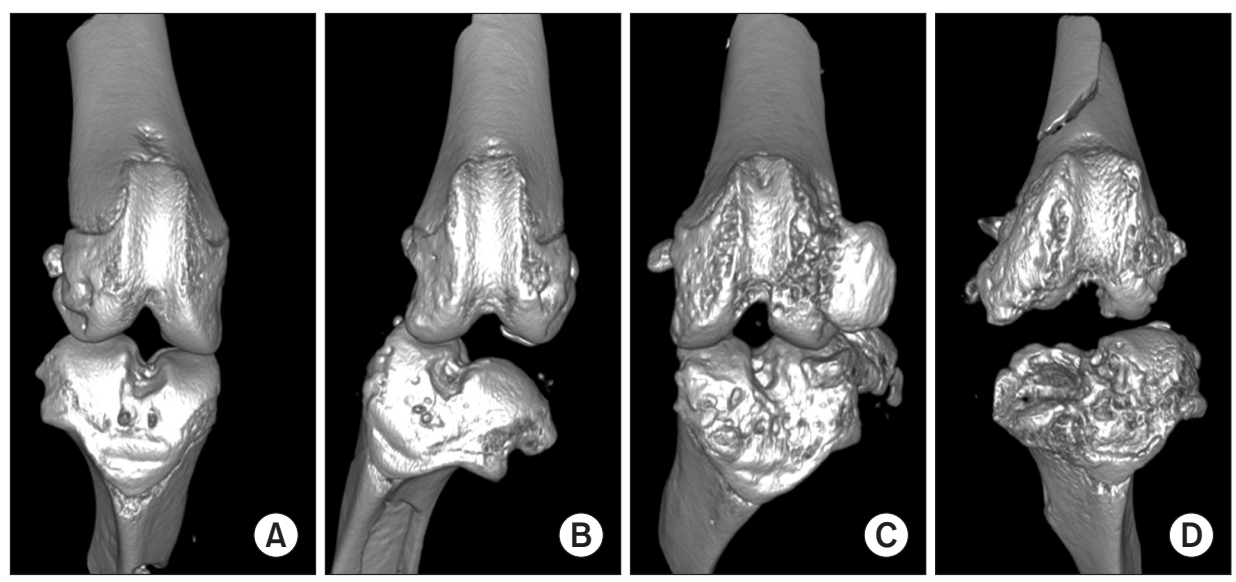

Fig. 4. Micro-computed tomography images of knee joints with three-dimensional reconstruction. Each image is from a different surgery group. (A) Sham surgery, (B) bilateral ovariectomy, (C) anterior cruciate ligament transection with medial meniscectomy, and (D) bilateral ovariectomy and anterior cruciate ligament transection with medial meniscectomy.

tion. The micro-CT scanner scanning time was adjusted to 0.21 seconds with a setting of $80 \mathrm{kVp}, 500 \mu \mathrm{A}$, and 30 calibrations. A 30.74- $\mathrm{mm}$ axial and trans-axial fields of view were acquired. Bone mineral density (BMD) was calculated from the micro-CT images of knee joint, at the medial femoral condyle area and the lateral femoral condyle area of each sample. Micro-CT images were also reconstructed in a three-dimensional image to show $\mathrm{OA}$ changes (Fig. 4), which was described in a previous study [14].

\section{Statistical analysis}

The SPSS 20.0 program for Windows (IBM, Armonk, NY, USA) was used for statistics. Because of the small sample size, nonparametric statistics were used. Repeated-measures ANOVA test was used to evaluate the behavioral tests (number of rears). Also, the Kruskal-Wallis test was used to examine if there were differences in the histologic study and in the bone mineral density values among each group. If the Kruskal-Wallis test rejected the null hypothesis, then post-hoc Mann-Whitney U test with Bonferroni corrected $\mathrm{p}$-value was performed. The significance level was defined as $\mathrm{p} \leq 0.008$ (Bonferroni corrected $\mathrm{p}$-value).

All experimental procedures were performed in accordance with standard operating protocols established by the Institutional Animal Care and Use Committee (IACUC) at our biomedical research institute. All protocols in this study were approved by the Institutional Review Board of Animal Experiments at Samsung Medical Center (Approval code: K-B2-001, 2011).

\section{RESULTS}

\section{Evaluation of behavioral tests (number of rears)}

At 1 week, no significant differences in the number of rears was found across each group. In the SHAM group, there was no change in the number of rears at 1,2, and 4 weeks. In the OVX group, the number of rears remained unchanged at 1 and 2 weeks, but it decreased at 4 weeks. Continuous decrease in the number of rears at 1,2 , and 4 weeks was observed in the ACLT with MM group and the OVX plus ACLT with MM group. Although there was no significant difference, the number of rears in the OVX plus ACLT with MM group showed a more downward trajectory with time than in the ACLT with MM group (Fig. 5). Repeated-measures ANOVA testing revealed that there was a significant temporal change in the number of rears for the overall groups $(\mathrm{F}=7.181, \mathrm{df}=1, \mathrm{p}=0.016)$. However, there was no significant difference between the groups in the number of rears $(\mathrm{F}=0.744, \mathrm{df}=3, \mathrm{p}=0.542)$.

\section{Histologic study}

Modified Mankin's scores were $1.8 \pm 0.4$ in the SHAM group, $5.2 \pm 1.3$ in the OVX group, $7.2 \pm 0.8$ in the ACLT with MM group, and 9.8 \pm 1.8 in the OVX plus ACLT with MM group (Fig. 6). There were significant differences among all groups in the Kruskal-Wallis test $(\mathrm{p}=0.001)$. MannWhitney U test with Bonferroni correction revealed that OVX plus ACLT with MM group had a significantly higher modified Mankin's score than OVX group $(\mathrm{p}=0.008)$ and SHAM group $(\mathrm{p}=0.008)$.

Micro-CT imaging study

BMDs of 19 rats were calculated at the medial femoral 


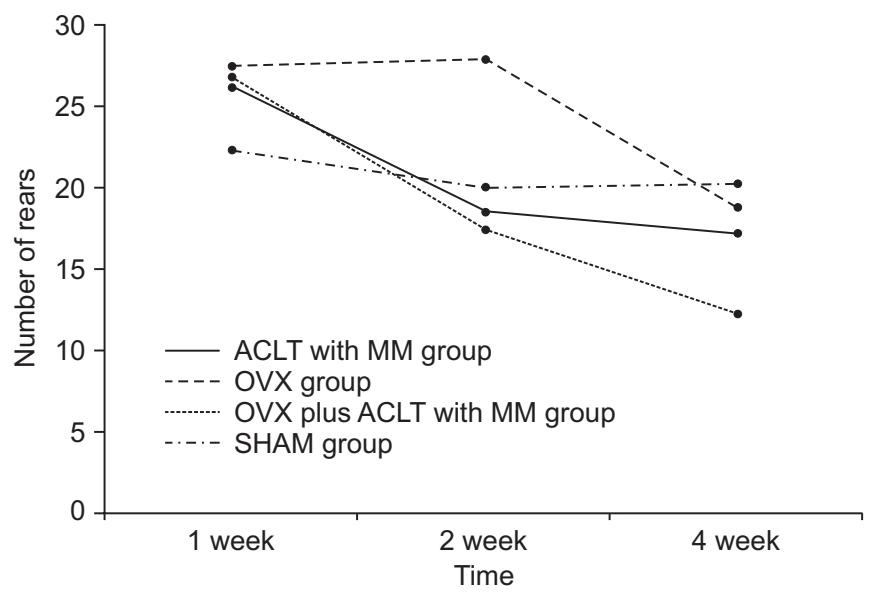

Fig. 5. The number of rears at 1,2 , and 4 weeks after surgery in the sham surgery group (SHAM group), the bilateral ovariectomy group (OVX group), the anterior cruciate ligament transection with medial meniscectomy group (ACLT with MM group), and the bilateral ovariectomy plus anterior cruciate ligament transection with medial meniscectomy group (OVX plus ACLT with MM group). This figure shows significant decrease in the number of rears for the overall groups ( $\mathrm{p}<0.05$ for time effect), but there was no significant difference between the groups in the number of rears $(\mathrm{p}=0.542)$.

condyle area and the lateral femoral condyle. The mean BMDs for the SHAM group, the OVX group, the ACLT with MM group, and the OVX plus ACLT with MM group were 862, 828, 859 and 723, respectively (Fig. 7). The KruskalWallis scores were significantly different between the groups $(\mathrm{p}=0.004)$. In post-hoc analysis, there was a significant difference of BMDs between the SHAM group, and the OVX plus ACLT with MM group ( $\mathrm{p}=0.003$ ). In addition, significant differences for BMDs existed between the ACLT with MM group, and the OVX plus ACLT with MM group ( $\mathrm{p}=0.002)$.

\section{DISCUSSION}

From this study, we found that the model of bilateral OVX plus ACLT with MM displayed phenotypes of OA for histology and BMD, phenotypes that were derived from contributions of bilateral OVX and ACLT with MM alone. What is known is that bilateral OVX might cause compositional changes in cartilage and subchondral bone (osteoporotic change) and ACLT with MM might lead to structural destruction by joint instability, and combination of these two procedures could lead to more charac-

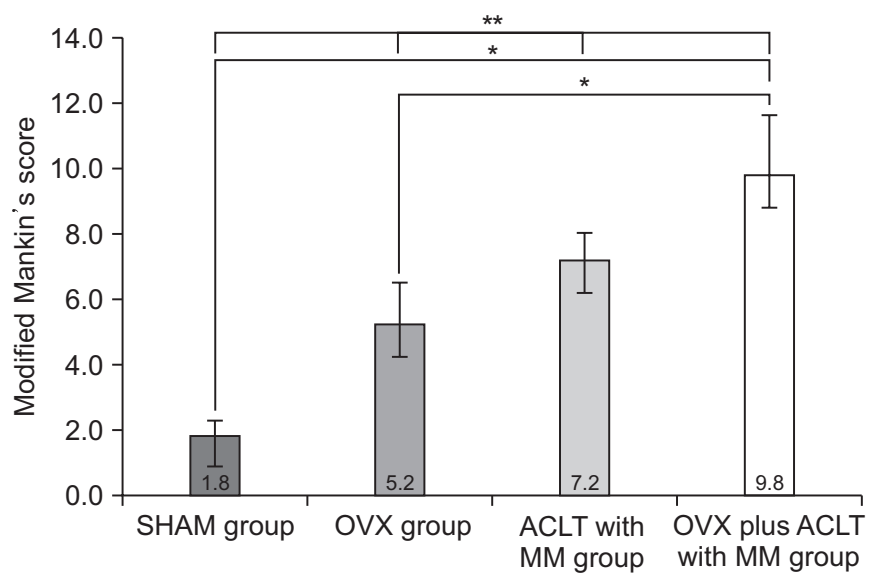

Fig. 6. Modified Mankin's scores of the sham surgery group (SHAM group), the bilateral ovariectomy group (OVX group), the anterior cruciate ligament transection with medial meniscectomy group (ACLT with MM group), and the bilateral ovariectomy plus anterior cruciate ligament transection with medial meniscectomy group (OVX plus ACLT with MM group). This figure shows significant differences among all groups $\left({ }^{* *} \mathrm{p}<0.001\right)$. OVX plus ACLT with MM group showed significantly higher modified Mankin's score than the OVX group $\left({ }^{*} \mathrm{p}=0.008\right)$ and the SHAM group $\left({ }^{*} \mathrm{p}=0.008\right)$. The Kruskal-Wallis test followed by post-hoc Mann-Whitney U test with Bonferroni correction.

teristic changes typical of OA and osteoporosis compared to single procedure alone (either bilateral OVX or ACLT with MMT).

Until now, only one study combined ACLT, MM, and OVX at the same time for OA animal models [15]. They used ACLT combined with the OVX animal model, in a model similar to ours to demonstrate the preventive effect of BIS076, a natural porcine cartilage extract on progression of OA. However, the authors did not demonstrate the combination effect of ACLT with MM plus OVX by comparing the ACLT and OVX animal models separately.

In the evaluation of behavioral tests, there was no remarkable change in the number of rears in the SHAM group; however, the number of rears decreased in other groups. Interestingly, in the OVX group, the number of rears at 1 and 2 weeks were similar, but at 4 week, the number decreased. This suggests that the estrogen levels are probably maintained for a period after OVX, but as the estrogen levels are further depleted, the injury to the joint progresses and this affects the rat's behavioral pattern. On the other hand, in the ACLT group, there was a 


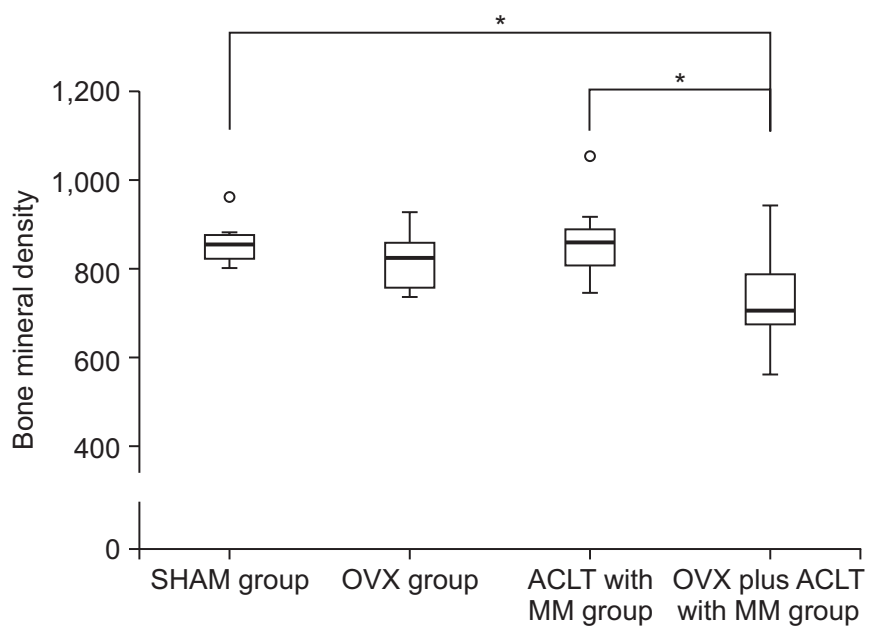

Fig. 7. Bone mineral density of the sham surgery group (SHAM group), the bilateral ovariectomy group (OVX group), the anterior cruciate ligament transection with medial meniscectomy group (ACLT with MM group), and the bilateral ovariectomy plus anterior cruciate ligament transection with medial meniscectomy group (OVX plus ACLT with MM group). There was a significant difference of bone mineral densities between not only the SHAM group and the OVX plus ACLT with MM group ( $\left.{ }^{*} \mathrm{p}=0.003\right)$ but also the ACLT with MM group and the OVX plus ACLT with MM group $\left({ }^{*} p=0.002\right)$. Small circle $(\bigcirc)$ is outlier. The Kruskal-Wallis test followed by post-hoc MannWhitney U test with Bonferroni correction.

large decrease in the number rears between week 1 and 2 . It is then presumed that as the ACLT procedure induces immediate and direct injury to the joint, this affects the behavioral pattern of the rat more quickly, unlike the continual injury observed in clinical OA. Rats with bilateral ACLT with MM and OVX had the biggest decrease in the number of rears.

Results of the present study correspond with the earlier study which reported that in a rabbit model, ovariohysterectomy altered cell metabolism in the connective tissues of the knee [16]. It appears that OVX accelerates the progression of OA change and aggravates pain. Therefore we postulate that combination of osteoporotic change from OVX and direct joint injury from ACLT creates a synergistic effect. The results in our study, however, were not statistically significant, as this might have been be due to the small sample size. If sample size had been larger, it could have added the necessary statistical significance for a meaningful conclusion.

From the histologic study, the damage to the cartilage tissues in each group was evaluated using the modified Mankin's score. In the order of the least to the highest severity, these scores corresponded with the SHAM group, the OVX group, the ACLT with MM group, and the OVX plus ACLT with MM group, respectively. This suggests that the instability and the lack of cushioning at the joint brought about by ACLT with MM is more detrimental to the articular cartilage than the osteoporotic changes caused by OVX, and the impact to the joint is maximal when OVX is combined with ACLT with MM. These results are consistent with previous data, suggesting that presence of estrogen may have an inhibitory and prophylactic effect on cartilage degeneration and thus maintain the health of the cartilage [17].

The decrease in BMD and the resulting osteoporosis are frequently seen in postmenopausal women [18], as the estrogen levels from the ovaries decrease to low levels post menopause. This is also seen with oophorectomy. The reduced estrogen levels in both cases reduce the rates of bone remodeling and lead to conditions of osteoporosis [19].

The prevalence of $\mathrm{OA}$ is also increased after menopause [20]. However the relationship between BMD changes from osteoporosis to the onset of $\mathrm{OA}$ is unclear. According to one review and from a number of systematic, crosssectional studies, occurrence of OA and osteoporosis are inversely related, but the BMD for bones around the OAaffected joint is decreased [21]. According to the Framingham study, in women with osteophytosis of the knee, femoral BMD was higher and joint space narrowing was not significantly associated with BMD [22]. However, in our results, the mean of femoral condyle BMD in the OVX plus ACLT with MM group was lower than that of other groups. These results are in agreement with a previous study, reporting a decrease in periarticular subchondral BMD in mild OA patients [23]. In addition, Lee et al. [24] reported that BMD loss was associated with progressive cartilage loss in knees with OA. Overall, it is inferred that OVX-induced osteoporosis and ACLT with MM-induced cartilage damage led to BMD decrease.

Unexpectedly, a significant BMD difference was not observed in the OVX group, but only for the OVX plus ACLT with MM group. This was different from the study by Inada et al. [25], who investigated the relationship between bone tissues and ovariectomy. The reason for the contradictory results might be due to the fact that 
a 4-week study was too short to evaluate bone mineral change from OVX alone, but enough to evaluate the bone mineral change from OVX and ACLT with MM as the unstable stress triggered by ACLT with MM further damaged the weakened cartilage caused by OVX. In a previous study, the femur BMD in the OVX group was not significantly decreased in 4 weeks after OVX, but significantly decreased in 8 weeks after OVX compared with that for the SHAM group [26].

This study had the limitation of a small sample size. The lack of significance for the number of rears may have been due to the small sample size and could have improved with more animals per group. Despite this, the change in the number of rears at 4 weeks was 14.6 in OVX plus ACLT with MM group but was only 2.1 in the SHAM group, indicating a large effect. Further studies are required with a larger test group to reach significance.

In summary, we found that the bilateral OVX plus ACLT with MMT rat model had changes in terms of histology and BMD more indicative of OA than compared to either the bilateral OVX model or the ACLT with MMT model. Although the behavioral test did not reveal significant differences between the groups due to the small sample size, histological data and BMD showed significant differences for OVX and ACLT with MM compared with OVX or ACLT with MM alone. Therefore, the OVX and ACLT with MM model demonstrated to be an appropriate degenerative OA animal model in our study.

\section{CONFLICT OF INTEREST}

No potential conflict of interest relevant to this article was reported.

\section{ACKNOWLEDGMENTS}

This study was supported by the Samsung Medical Center-KIST Transitional Research Grant (No. SMX1150031) and by the National Research Foundation of Korea (No. 2013R1A1A1007404).

\section{REFERENCES}

1. Spector TD, Hart DJ, Byrne J, Harris PA, Dacre JE, Doyle DV. Definition of osteoarthritis of the knee for epidemiological studies. Ann Rheum Dis 1993;52:790-4.
2. Little CB, Hunter DJ. Post-traumatic osteoarthritis: from mouse models to clinical trials. Nat Rev Rheumatol 2013;9:485-97.

3. Poole R, Blake S, Buschmann M, Goldring S, Laverty S, Lockwood S, et al. Recommendations for the use of preclinical models in the study and treatment of osteoarthritis. Osteoarthritis Cartilage 2010;18 Suppl 3:S10-6.

4. Pickarski M, Hayami T, Zhuo Y, Duong LT. Molecular changes in articular cartilage and subchondral bone in the rat anterior cruciate ligament transection and meniscectomized models of osteoarthritis. BMC Musculoskelet Disord 2011;12:197.

5. Sniekers YH, Weinans H, Bierma-Zeinstra SM, van Leeuwen JP, van Osch GJ. Animal models for osteoarthritis: the effect of ovariectomy and estrogen treatment. A systematic approach. Osteoarthritis Cartilage 2008;16:533-41.

6. Zhang Y, McAlindon TE, Hannan MT, Chaisson CE, Klein R, Wilson PW, et al. Estrogen replacement therapy and worsening of radiographic knee osteoarthritis: the Framingham Study. Arthritis Rheum 1998;41:1867-73.

7. Turner AS, Athanasiou KA, Zhu CF, Alvis MR, Bryant HU. Biochemical effects of estrogen on articular cartilage in ovariectomized sheep. Osteoarthritis Cartilage 1997;5:63-9.

8. Reis EM, Ropke J, Busanello A, Reckziegel P, Leal CQ, Wagner C, et al. Effect of Hypericum perforatum on different models of movement disorders in rats. Behav Pharmacol 2013;24:623-7.

9. Fowler SC, Muma NA. Use of a force-sensing automated open field apparatus in a longitudinal study of multiple behavioral deficits in CAG140 Huntington's disease model mice. Behav Brain Res 2015;294:7-16.

10. Nagase H, Kumakura S, Shimada K. Establishment of a novel objective and quantitative method to assess pain-related behavior in monosodium iodoacetateinduced osteoarthritis in rat knee. J Pharmacol Toxicol Methods 2012;65:29-36.

11. Kuroki H, Nakagawa Y, Mori K, Ohba M, Suzuki T, Mizuno Y, et al. Acoustic stiffness and change in plug cartilage over time after autologous osteochondral grafting: correlation between ultrasound signal intensity and histological score in a rabbit model. Arthritis Res Ther 2004;6:R492-504. 
12. Ostergaard K, Andersen CB, Petersen J, Bendtzen K, Salter DM. Validity of histopathological grading of articular cartilage from osteoarthritic knee joints. Ann Rheum Dis 1999;58:208-13.

13. Pauli C, Whiteside R, Heras FL, Nesic D, Koziol J, Grogan SP, et al. Comparison of cartilage histopathology assessment systems on human knee joints at all stages of osteoarthritis development. Osteoarthritis Cartilage 2012;20:476-85.

14. Chan WP, Lang P, Stevens MP, Sack K, Majumdar S, Stoller DW, et al. Osteoarthritis of the knee: comparison of radiography, CT, and MR imaging to assess extent and severity. AJR Am J Roentgenol 1991;157:799806.

15. Ferrandiz ML, Terencio MC, Carceller MC, Ruhí R, Dalmau P, Verges J, et al. Effects of BIS076 in a model of osteoarthritis induced by anterior cruciate ligament transection in ovariectomised rats. BMC Musculoskelet Disord 2015;16:92.

16. Hart DA, Achari Y. Alterations to cell metabolism in connective tissues of the knee after ovariohysterectomy in a rabbit model: are there implications for the postmenopausal athlete? Br J Sports Med 2010;44:86771

17. Yoshida A, Morihara T, Matsuda K, Sakamoto H, Arai Y, Kida Y, et al. Immunohistochemical analysis of the effects of estrogen on intraarticular neurogenic inflammation in a rat anterior cruciate ligament transection model of osteoarthritis. Connect Tissue Res 2012;53:197-206.

18. Finkelstein JS, Brockwell SE, Mehta V, Greendale GA,
Sowers MR, Ettinger B, et al. Bone mineral density changes during the menopause transition in a multiethnic cohort of women. J Clin Endocrinol Metab 2008;93:861-8.

19. Lindsay R, Hart DM, Sweeney A, Coutts JR, Clarke A. Endogenous oestrogen and bone loss following oophorectomy. Calcif Tissue Res 1977;22 Suppl:213-6.

20. Martin-Millan M, Castaneda S. Estrogens, osteoarthritis and inflammation. Joint Bone Spine 2013;80:36873.

21. Im GI, Kim MK. The relationship between osteoarthritis and osteoporosis. J Bone Miner Metab 2014;32:1019.

22. Hannan MT, Anderson JJ, Zhang Y, Levy D, Felson DT. Bone mineral density and knee osteoarthritis in elderly men and women: the Framingham study. Arthritis Rheum 1993;36:1671-80.

23. Karvonen RL, Miller PR, Nelson DA, Granda JL, Fernandez-Madrid F. Periarticular osteoporosis in osteoarthritis of the knee. J Rheumatol 1998;25:2187-94.

24. Lee JY, Harvey WF, Price LL, Paulus JK, DawsonHughes B, McAlindon TE. Relationship of bone mineral density to progression of knee osteoarthritis. Arthritis Rheum 2013;65:1541-6.

25. Inada M, Matsumoto C, Miyaura C. Animal models for bone and joint disease: ovariectomized and orchidectomized animals. Clin Calcium 2011;21:164-70.

26. Lei Z, Xiaoying Z, Xingguo L. Ovariectomy-associated changes in bone mineral density and bone marrow haematopoiesis in rats. Int J Exp Pathol 2009;90:512-9. 